\title{
Effect of Esmolol, Labetalol and Metoprolol for Attenuating the Cardiovascular Stress Response to Laryngoscopy and Intubation: A Comparative Study
}

\author{
Deepak $\mathbf{R}^{1}$, Jaya Lalwani ${ }^{2}$, Prathibha Jain Shah², K P Dubey ${ }^{2}$ \\ ${ }^{1}$ Assistant Professor, Department of Anaesthesia, ESIC Medical College \& PGIMSR, K K Nagar, Chennai 600078, India, ${ }^{2}$ Professor, Department of \\ Anaesthesia, Pt.Jawaharlal Nehru Memorial Medical College, Raipur, Chhattisgarh, India.
}

\section{Abstract}

Background: To compare esmolol, labetalol and metoprolol in attenuating the cardiovascular response of L\&I. Subjects and Methods: It was a randomized prospective study in 120 patients of 18-60 years, of ASA grade I and II, of either sex, posted for elective surgery under GA. After approval from ethical committee and informed written consent, the patients were randomly allocated in four groups of 30 each. Group C (control) received $10 \mathrm{ml} 0.9 \%$ saline, group E esmolol $0.5 \mathrm{mg} / \mathrm{kg}$ (both $2 \mathrm{~min}$ prior to induction), Group L labetalol $0.25 \mathrm{mg} / \mathrm{kg}$ and Group M metoprolol $0.1 \mathrm{mg} / \mathrm{kg}$ (both $5 \mathrm{~min}$ prior to induction). All patients were pre-medicated with inj. ondansetron $0.1 \mathrm{mg} / \mathrm{kg}$, inj. glycopyrrolate $0.004 \mathrm{mg} / \mathrm{kg}$, inj. pen-tazocine $0.6 \mathrm{mg} / \mathrm{kg}$ and inj. midazolam $1 \mathrm{mg}$. All patients were induced with inj. thiopentone $5 \mathrm{mg} / \mathrm{kg}$ and succinylcholine $2 \mathrm{mg} / \mathrm{kg}$. Anaesthesia was maintained on isoflurane, $\mathrm{O} 2 \mathrm{~N} 2 \mathrm{O}$ and Atracurium. Heart rate and BP were recorded: pre-operative, after pre-medication, after induction, after L\&I, after 1, 3, 5, 10, 15 minutes following L\&I. Results: All the study drugs significantly attenuated the HR, SBP, DBP, MBP and RPP following L\&I compared to control. Metoprolol attenuated the heart rate and RPP compared to esmolol and labetalol. Esmolol attenuated the heart rate immediately following L\&I better than labetalol and significantly attenuated the SBP at $5 \mathrm{~min}$ and $10 \mathrm{~min}$ following L\&I. All readings of RPP were lower in esmolol in comparison to labetalol. Esmolol, was better than labetalol in attenuating the hemodynamic response. Sinus tachycardia and hypotension were the common side effects. Three patients in control and one in labetalol group developed ectopic beats following L\&I. One patient in esmolol had pain on i.v injection. Conclusion: Metoprolol attenuated the cardiovascular stress response to L \& I in comparison to esmolol and labetalol. Esmolol was comparably better than labetalol. Metoprolol can be used as alternative to esmolol and labetalol.

Keywords: Esmolol, Labetalol, Metoprolol.

Corresponding Author: Dr Deepak R, D26, ESIC Staff Quarters, 143 Sterling Road, Next to ES-IC Regional Office, Tamilnadu Nungambakkam, Chennai 600034

Received: June 2019

Accepted: July 2019

\section{Introduction}

Laryngoscopy and endotracheal intubation is an indispensable part of prac-tice for an anaesthesiologists career despite the advent of technological advances in airway equipment. L\&I causes cardiovascular changes which are largely ignored or taken for granted.

These responses were initially described as reflex in nature [King BD], ${ }^{[18]}$ but were stated to be vasovagal type or as being the result of reflex sympatho-adrenal stimulation [Bruder $\mathrm{N}]{ }^{[2]}$ caused by the efferent responses from the pharyngeal stimulation. There is increase in heart rate, blood pres-sure [Forbes AM, Prys Roberts C, Stoelting RK], ${ }^{[10,31,43]}$ intracranial pressure and intraocular pressure. There is an average increase in blood pressure by $40-50 \%$ and $20 \%$ increase in heart rate. ${ }^{[2]}$

It is believed that the increase in the arterial blood pressure during L\&I is predominantly due to an increase in cardiac output and less predominantly due to increase in SVR. There is an associated increase in CVP and some-times arrhythmias.

These cardiovascular stress responses can be detrimental in patients of cardiovascular diseases like hypertension, coronary artery diseses, and in CNS conditons of raised ICP$\mathrm{EDH}, \mathrm{SDH}$, aneurysms, intracranial tumors etc. ${ }^{[10]}$

LVF, MI, cerebral haemorrhage can occur in susceptible patients. Convul-sions can occur in parturients with preeclampsia.

Esmolol is an ultrashort acting beta-blocker with rapid onset of action. Its elimination half life is $9.2 \mathrm{~min}$. It is metabolized by red cell esterases into methanol and other inactive metabolites. Esmolol achieves peak effect on heart rate within one minute and on blood pressure within two minute of i.v injection [Miller Donald R]. ${ }^{[28]}$

Labetalol is a combined alpha 1 and beta blocker. It has on onset of action of $5 \mathrm{~min}$. Its average duration of action is 6 hrs. The i.v dose is $10-20 \mathrm{mg}$ given over $2 \mathrm{~min}$, followed by 
0

repeat dose every $10 \mathrm{~min}$ till the clinically desired BP is attained.

Metoprolol is a beta-1 selective adrenoreceptor blocker. It decreases heart rate and contractility leading to decrease in cardiac output. Given in a dose of $0.1 \mathrm{mg} / \mathrm{kg}$, the maximum response is obtained in $20 \mathrm{~min}$ on iv injection and the duration of action is about 4 hours.

We did a study to assess and compare esmolol, labetalol and metoprolol in attenuating the cardiovascular response of L\&I.

\section{Aims and objectives}

The Aims and objectives of the study is to assess and compare the effects of esmolol, labetalol and metoprolol in attenuating the haemodynamic cardi-ovascular pressor response in L\&I and to evaluate for any side effects intra or perioperatively.

\section{Subjects and Methods}

The present study was conducted in the Department of Anaesthesiology and Critical Care, Pt. J.N.M. Medical College and Dr. B.R.A.M. Hospital Raipur, Chhattisgarh after approval from ethical committee.

It was a prospective, randomised controlled study. We compared esmolol, labetalol and metoprolol in decreasing the cardiovascular stress response during rigid laryngoscopy and intubation.

The study included 120 normotensive patients (30 in each group)belonging to ASA grade I and II of either sex undergoing elective surgery requiring gen-eral anaesthesia and intubation in general surgery, ENT, orthopaedics and gynaecological procedures.

All the patients were randomly allocated into four groups of 30 each to re-ceive the study drugs:-

1. Group C: $0.9 \%$ saline $10 \mathrm{ml}$ given as control

2. Group E: iv esmolol $0.5 \mathrm{mg} / \mathrm{kg}$

3. Group L: slow iv labetalol $0.25 \mathrm{mg} / \mathrm{kg}$

4. Group M: slow iv metoprolol $0.1 \mathrm{mg} / \mathrm{kg}$

All the study drugs were diluted to $10 \mathrm{ml} 0.9 \% \mathrm{NS}$.

\section{Criteria for selection of patients}

Inclusion criteria

i. ASA physical status I and II adult patients

ii. Age 18-45 years

iii. Either sex

iv. Normotensive patient

\section{Exclusion criteria}

i. Cardiovascular diseases

a. Hypertension

b. Ischemic heart disease

c. Recent myocardial infarction

d. Cardiac failure

e. Sinus bradycardia $(<60 /$ min or heart block $)$

f. Current treatment with $\beta$-blockers, verapamil, diltiazem and amiodarone

ii. Pulmonary diseases: Chronic obstructive airway disease or asthma iii. Hepatic diseases

iv. Renal diseases

v. Patients with anticipated difficult airway; laryngoscopy and intubation time more than 30 seconds or requiring more than two attempts

vi. Poor general condition

vii. Fever(temp.>99 deg. F)

viii. Diabetes mellitus

ix. Anaemia with $\mathrm{Hb}<10 \mathrm{~g} / \mathrm{dl}$.

\section{Pre-operative Assessment}

A detailed pre-operative assessment of the patient was done after taking complete history, clinical examination and recording of vital parameters.

Informed written consent was taken from all the patients.

Following investigations were carried out in all patients:-

1. Hb, TLC, DLC, ESR

2. Urine examination routine and microscopy

3. Blood glucose level fasting and post prandial

4. Blood urea

5. Chest X-ray PA view

6. $\mathrm{ECG}$

7. Other investigations were carried out if indicated

\section{Protocol}

1. All the patients were kept fasting 6 hours preoperatively.

2. In the operation theatre, on day of surgery the patients were again ex-amined. Pre-induction (baseline) HR, SBP, DBP and MBP were noted. ECG monitoring was done with Multipara monitors.

3. Intravenous access was secured with $18 \mathrm{G}$ i.v cannula with RL.

4. Pre-anaesthetic medication was done15 minutes prior to induction with:

a. Inj. Ondansetron $\sim 4 \mathrm{mg}(0.1 \mathrm{mg} / \mathrm{kg})$

b. Inj. Glycopyrrolate $\sim 0.2 \mathrm{mg}(0.004 \mathrm{mg} / \mathrm{kg})$

c. Inj. Pentazocine $0.6 \mathrm{mg} / \mathrm{kg}$

d. Inj. Midazolam $1 \mathrm{mg}$

5. Injection of the study drugs and saline:-

i. In group $\mathrm{C}$, received $10 \mathrm{ml}$ of $0.9 \%$ saline $2 \mathrm{~min}$ before L\&I.

ii. In group E, $0.5 \mathrm{mg} / \mathrm{kg}$ of esmolol was given $2 \mathrm{~min}$ prior to L\&I

iii. In group L, $0.25 \mathrm{mg} / \mathrm{kg}$ of labetalol was given $5 \mathrm{~min}$ prior to L\&I.

iv. In group M, $0.1 \mathrm{mg} / \mathrm{kg}$ of metoprolol was given $5 \mathrm{~min}$ prior to L\&I.

All the study drugs were diluted to $10 \mathrm{ml} 0.9 \%$ normal saline (Q.S)

6. Pre-oxygenation was done with $100 \%$ for $3-5$ minutes.

7. Induction:

i. All patients were induced with inj. thiopentone 5 $\mathrm{mg} / \mathrm{kg}$ followed by suc-cinylcholine $2 \mathrm{mg} / \mathrm{kg}$ to facilitate intubation.

ii. After maximal relaxation and IPPV, laryngoscopy was carried out by Ma-cintosh laryngoscope and intubation was achieved with appropriate size cuffed orotracheal tube. Duration of laryngoscopy and number of attempts required for intubation were noted.

8. Maintenance: Anaesthesia was maintained on isoflurane; 
O2, N2O: 40:60 and inj Atracurium and IPPV.

9. Reversal: At the end of surgery, reversal was done with inj. neostigmine $(40 \mu \mathrm{g} / \mathrm{kg})$ and inj. glycopyrrolate $(0.008$ $\mathrm{mg} / \mathrm{kg}$ )

\section{Monitoring}

Haemodynamic monitoring: Heart rate, systolic blood pressure, diastolic blood pressure, mean blood pressure, respiratory rate and oxygen saturation $(\mathrm{SpO} 2)$ and ECG changes were monitored at various time intervals.

i. Pre-operative

ii. After pre-medication

iii. After induction

iv. Just after laryngoscopy and intubation

v. After $1 \mathrm{~min}, 3 \mathrm{~min}, 5 \mathrm{~min}, 10 \mathrm{~min}, 15 \mathrm{~min}$ following L\&I

Rate pressure product was derived and recorded at the same time intervals.

\section{Haemodynamic changes:}

i. Heart rate below 50 beats per minute was considered as bradycardia.

ii. Heart rate above 120 beats per minute was considered as sinus tachy-cardia.

iii. Systolic Blood Pressure below 90mmHg was considered as hypotension.

iv. Fall in SpO2 below $90 \%$ and any signs of respiratory distress were con-sidered significant and treated.

\section{Analysis of results and statistical methods:}

The results were analyzed by various statistical techniquespercentage, mean and standard deviation

\section{Probability value (P value):-}

1. Significance of difference between means within a group i.e comparison of the haemodynamic variations with their respective baseline values was calculated by paired t-test.

2. Significance of difference between means of the groups was found out by ANOVA test (analysis of variance).

A 'p' value $<0.05$ was taken as significant.

All the data were compiled in masterchart, tabulated, calculated and ana-lysed with the help of Figure-pad prism software.

\section{Results}

The observations recorded in each group are shown in the following tables and Figures:

\section{Table 1: Drug Distribution}

\begin{tabular}{|l|l|l|}
\hline Drug & No. of Cases & Group \\
\hline Control & 30 & C \\
\hline Esmolol & 30 & E \\
\hline Labetalol & 30 & L \\
\hline Metoprolol & 30 & M \\
\hline
\end{tabular}

[Table 1] shows the distribution of cases according to the drug used [Figure 1]. Patients were randomly divided into four groups with 30 patients in each group.

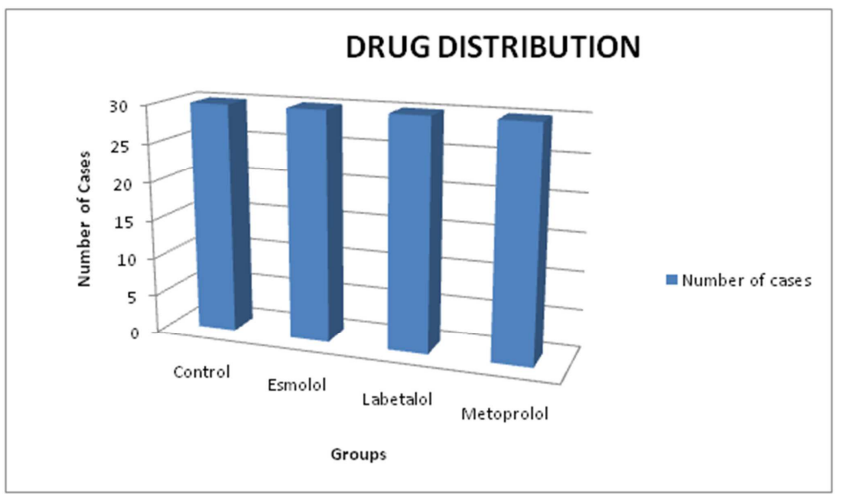

Figure 1: Drug Distribution

\begin{tabular}{l|l|l|l|l|}
\hline Table 2: Doses of Drug Received \\
\hline & Group C & Group E & Group L & Group M \\
\hline $\begin{array}{l}\text { Dose of the drug } \\
\text { recieved }\end{array}$ & $0.9 \%$ NS & $0.5 \mathrm{mg} / \mathrm{kg}$ & $0.25 \mathrm{mg} / \mathrm{kg}$ & $0.1 \mathrm{mg} / \mathrm{kg}$ \\
\hline
\end{tabular}

[Table 2] shows the doses of the drug received $(\mathrm{mg} / \mathrm{kg})$ by the cases [Figure 2] Group C received 0.9\% normal saline $10 \mathrm{ml}$, group E received esmolol $0.5 \mathrm{mg} / \mathrm{kg}$, group L received labetalol $0.25 \mathrm{mg} / \mathrm{kg}$ and group $\mathrm{M}$ received meto-prolol $0.1 \mathrm{mg} / \mathrm{kg}$. Labetalol and metoprolol was given 5 minutes before induc-tion whereas saline and esmolol was given 2 min prior to induction. All the study drugs were diluted to 10 ml N.S (Q.S)

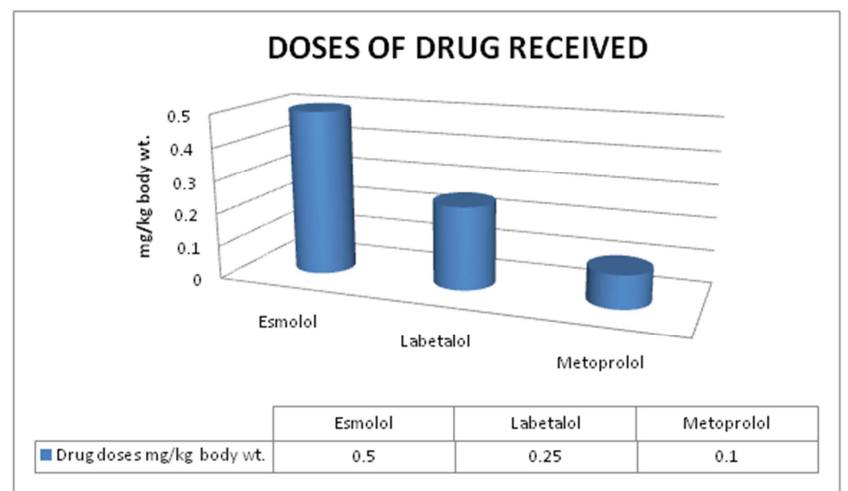

Figure 2: Doses of Drug Received

Table 3a: DemoFigureic Profile: Age And Body Weight Distri-

Bution

\begin{tabular}{|l|l|l|l|l|l|}
\hline S.No. & Variables & $\begin{array}{l}\text { Groupc } \\
(\text { Mean } \pm \\
\text { Sd) }\end{array}$ & $\begin{array}{l}\text { Group E } \\
(\text { Mean } \pm \\
\text { Sd) }\end{array}$ & $\begin{array}{l}\text { Group L } \\
\text { (Mean } \pm \\
\text { Sd) }\end{array}$ & $\begin{array}{l}\text { Group } \\
\text { M } \\
\text { (Mean } \\
\pm \text { Sd) }\end{array}$ \\
\hline 1. & Age (years) & $\begin{array}{l}37.57 \pm 9 . \\
67\end{array}$ & $35.53 \pm 11$. & $36.87 \pm 14$. & $35.87 \pm 1$ \\
& & 41 & 59 & 2.22 \\
\hline 2. & Weight $(\mathrm{kg})$ & $54.64 \pm 9$. & $55.37 \pm 11$. & $54.27 \pm 10$. & $56.23 \pm 1$ \\
& & 94 & 56 & 23 & 2.51 \\
\hline
\end{tabular}

[Table 3a] shows that the four groups are comparable with respect to age and weight [Figure 3a (i) and (ii)]. The groups were comparable with respect to age and weight. The mean age (in years) was $37 \pm 9.67,35.53 \pm 11.41,36.87 \pm 14.59$ and $35.87 \pm 12.22$ in the groups $\mathrm{C}, \mathrm{E}, \mathrm{L}$ and $\mathrm{M}$ respectively. The mean weight (in $\mathrm{kg}$ ) was $54.64 \pm 9.94,55.37 \pm 11.56$, $54.37 \pm 10.23$ and $56.23 \pm 12.51$ respectively. The youngest patient in all the groups was 18 years. The oldest patient in 

0 groups $\mathrm{E}, \mathrm{L}$ and $\mathrm{M}$ was 60 years while that in group $\mathrm{C}$ was 57 years.

Table 3b: DemoFigureic Profile: Sex Distribution

\begin{tabular}{|l|l|l|l|l|}
\hline Sex & Group C & Group E & Group L & Group M \\
\hline Male & 15 & 14 & 16 & 14 \\
\hline Female & 15 & 16 & 14 & 16 \\
\hline
\end{tabular}

[Table 3b] shows that the four groups are comparable with respect to sex dis-tribution [Figure $3 \mathrm{~b}$ ]. The male to female ratios were $15: 15,14: 16,16: 14$ and $14: 16$ in groups $\mathrm{C}, \mathrm{E}, \mathrm{L}$ and $\mathrm{M}$ respectively.

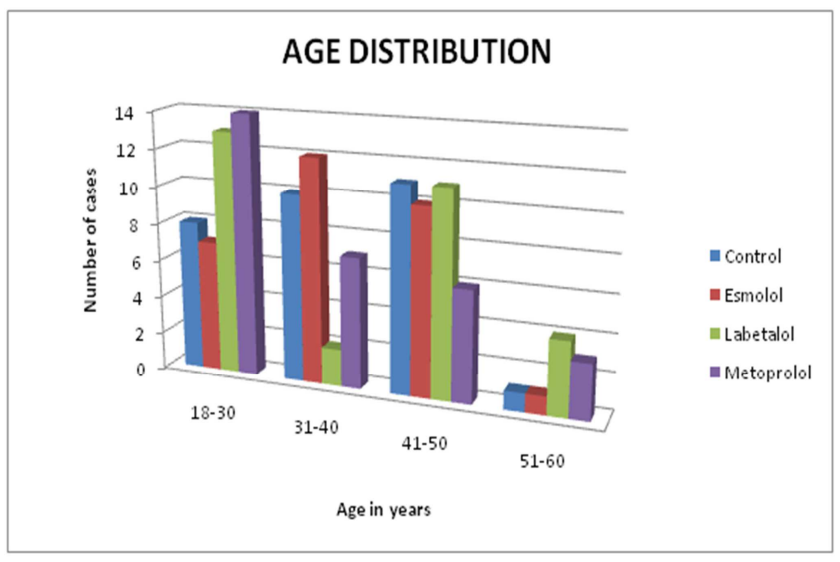

Figure 3a (i): Age Distribution

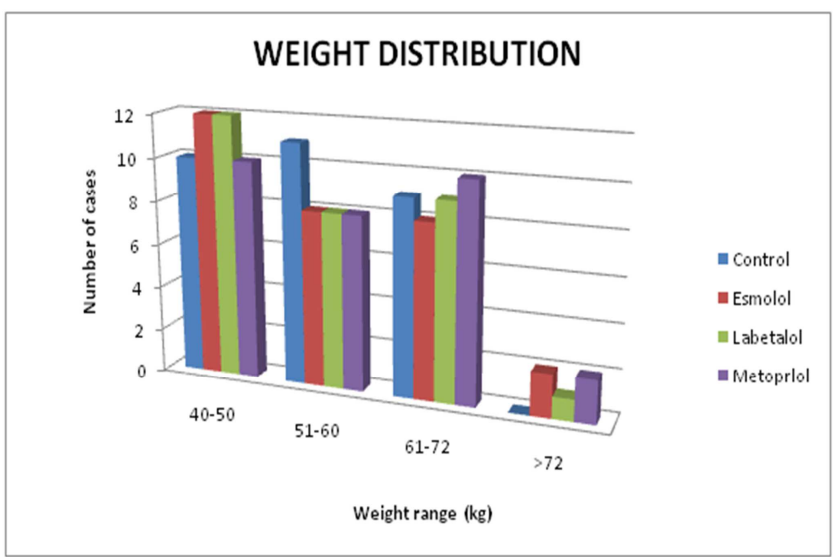

Figure 3a (ii): Weight Distribution

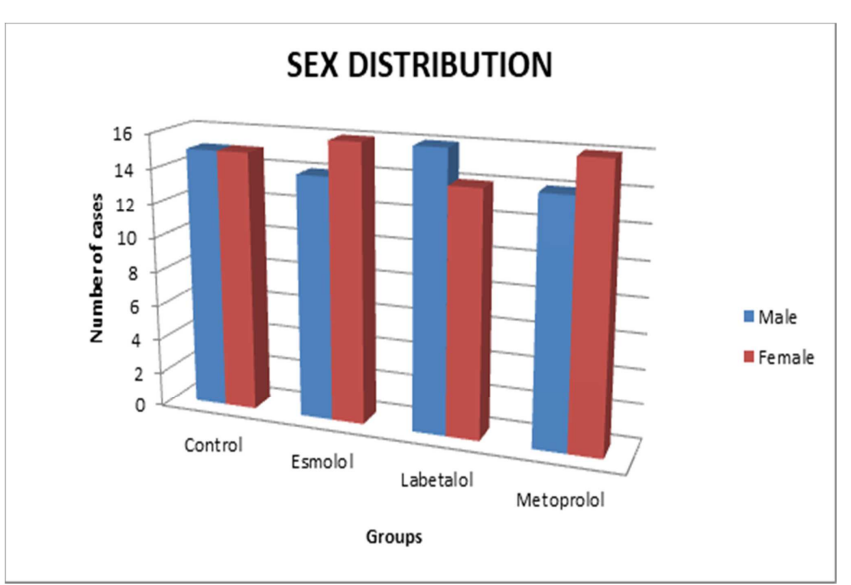

Figure 3b: Sex Distribution.
TABLE-4a

\begin{tabular}{|c|c|c|c|c|c|c|c|}
\hline & $\begin{array}{c}\text { GROUPC } \\
\text { MEAN士 SD } \\
\text { p compared to } \\
\text { pre-indn values }\end{array}$ & $\begin{array}{c}\text { GROUPE } \\
\text { MEAN } \pm \text { SD } \\
\text { p compared to } \\
\text { pre-indn.values }\end{array}$ & \begin{tabular}{|l|l}
$\begin{array}{l}\text { 'p' (compared } \\
\text { with control) }\end{array}$ \\
\end{tabular} & \begin{tabular}{|c|} 
GROUPL \\
MEAN士 SD \\
pcompared to \\
pre-indnvalues
\end{tabular} & $\begin{array}{l}\text { 'p' (compared } \\
\text { with control) }\end{array}$ & \begin{tabular}{|c|} 
GROUP M \\
MEAN士 SD \\
p compared to \\
pre-indn values
\end{tabular} & $\begin{array}{l}\text { 'p' (compared } \\
\text { with control) }\end{array}$ \\
\hline $\begin{array}{c}\text { Pre-induction } \\
\end{array}$ & $84.43 \pm 5.367$ & $87.77 \pm 7.267$ & $>0.05$ & \begin{tabular}{|c|}
$86.2 \pm 9.707$ \\
\end{tabular} & $>0.05$ & $88 \pm 8.94$ & $>0.05$ \\
\hline After induction & $\begin{array}{r}84.4 \pm 5.096 \\
p<0.0001\end{array}$ & $\begin{array}{c}87.43+7.704 \\
p<0.0001\end{array}$ & $>0.05$ & $\begin{array}{c}86.38+11.54 \\
p<0.0001\end{array}$ & $>0.05$ & $\begin{array}{c}86.33+7.009 \\
p=0.2925\end{array}$ & $>0.05$ \\
\hline Just after L\&1 & $\begin{array}{c}111.5+7.031 \\
p<0.0001\end{array}$ & $\begin{array}{l}103 \pm 7.531 \\
p<0.0001\end{array}$ & $<0.01$ & $\begin{array}{l}101.6 \pm 13.11 \\
p<0.0001\end{array}$ & $<0.0001$ & $\begin{array}{c}92.87 \pm 7.352 \\
p=0.0041\end{array}$ & $<0.0001$ \\
\hline $1 \mathrm{~min}$ after $L \& 1$ & $\begin{array}{c}108.7 \pm 6.215 \\
p<0.0001\end{array}$ & $\begin{array}{c}95.33+7.88 \\
p<0.0001\end{array}$ & $<0.0001$ & $\begin{array}{l}101 \pm 11.7 \\
p=0.0002\end{array}$ & $<0.01$ & $\begin{array}{c}92.37 \pm 7.266 \\
p=0.0055\end{array}$ & $<0.0001$ \\
\hline $3 \mathrm{~min}$ after $L \& 1$ & $\begin{array}{c}1028+4.942 \\
p<0.0001\end{array}$ & $\begin{array}{c}103.5+7.361 \\
p<0.0001 \\
0\end{array}$ & $>0.05$ & $\begin{array}{c}95.83+10.96 \\
p=0.0002 \\
p^{2}\end{array}$ & $<0.05$ & $\begin{array}{l}91.93 \pm 6.4 \\
p=0.0173\end{array}$ & $<0.00001$ \\
\hline $5 \mathrm{~min}$ after $L \& 1$ & $\begin{array}{c}101.4 \pm 4.582 \\
p<0.0001\end{array}$ & $\begin{array}{c}101.2 \pm 8.779 \\
p<0.0001\end{array}$ & $>0.05$ & $\begin{array}{c}95.57+11.96 \\
p=0.0005\end{array}$ & $>0.05$ & $\begin{array}{c}90.5 \pm 5.993 \\
p=0.0988\end{array}$ & $<0.0001$ \\
\hline 10 min after $1 \& \mid$ & $\begin{array}{c}94.83 \pm 5.2 .286 \\
p<0.0001\end{array}$ & $\begin{array}{c}95.23+7.4966 \\
p<0.0001\end{array}$ & $>0.05$ & $\begin{array}{l}91.9 \pm 10 \\
p=0.0245\end{array}$ & $>0.05$ & $\begin{array}{c}91.9 \pm 10 \\
83.73 \pm 8.851\end{array}$ & $<0.0001$ \\
\hline
\end{tabular}

Table 4 shows changes in the heart rate and comparison with baseline and control(Graph 4).

Table 4b: Changes In Heart Rate: Comparison among the Study Drug Groups

\begin{tabular}{|l|l|l|l|}
\hline $\begin{array}{l}\text { Heart Rate (beats/min) } \\
\text { w.r.t time }\end{array}$ & $\begin{array}{l}\text { Group E vs } \\
\text { L } \\
\text { P value }\end{array}$ & $\begin{array}{l}\text { Group L } \\
\text { vs M } \\
\text { P value }\end{array}$ & $\begin{array}{l}\text { Group M vs } \\
\text { E value } \\
\text { P val }\end{array}$ \\
\hline Pre-induction & $>0.05$ & $>0.05$ & $>0.05$ \\
\hline After induction & $>0.05$ & $>0.05$ & $>0.05$ \\
\hline Just after L \& I & $>0.05$ & $<0.01$ & $<0.001$ \\
\hline 1 min after L \& I & $>0.05$ & $<0.01$ & $>0.05$ \\
\hline 3 $\min$ after L\&I & $<0.05$ & $>0.05$ & $<0.0001$ \\
\hline 5 min after L \&I & $>0.05$ & $>0.05$ & $<0.0001$ \\
\hline 10 min after L\&I & $>0.05$ & $<0.01$ & $<0.0001$ \\
\hline
\end{tabular}

[Table 4b] shows comparison in heart rate among the study drug groups [Figure 4]. Metoprolol significantly attenuated the HR rise in comparison to labetalol just after L\&I, at $1 \mathrm{~min}$ and at $10 \mathrm{~min}(\mathrm{P}<0.01)$ and in comparison to esmolol just after L\&I, at $3 \mathrm{~min}, 5 \mathrm{~min}$ and $10 \mathrm{~min}(\mathrm{P}<0.0001)$. Labetalol significantly attenuated $(\mathrm{P}<0.05)$ the $\mathrm{HR}$ rise, $3 \mathrm{~min}$ after L\&I in comparison to esmolol.

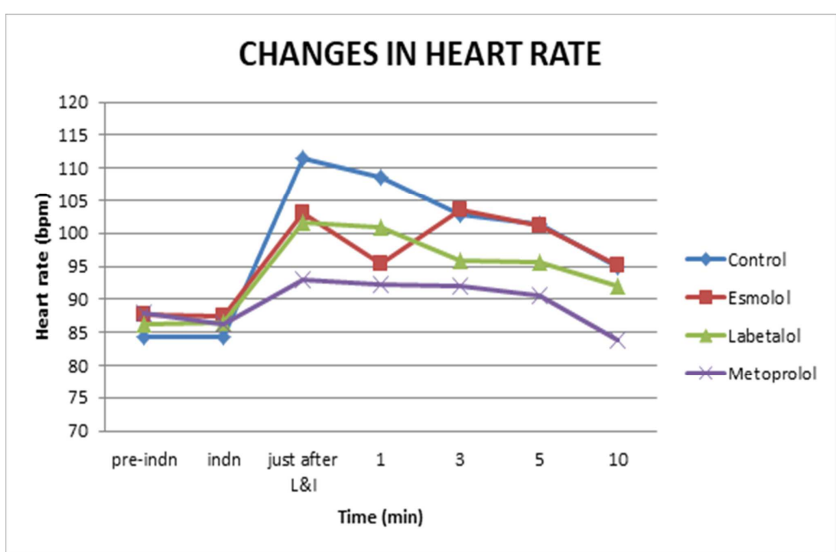

Figure 4: Changes in Heart Rate

TABLE-5a

CHANGES IN SYSTOLIC BLOOD PRESSURE: Comparison with Baseline and Control

\begin{tabular}{|c|c|c|c|c|c|c|c|}
\hline & GROUPC & GROUPE & & GROUPL & & GROUPM & \\
\hline & \begin{tabular}{c|} 
MEAN SD \\
p compared to \\
pre-indn values
\end{tabular} & \begin{tabular}{|c|} 
MEAN \pm SD \\
p compared to \\
pre-indn.values \\
\end{tabular} & $\begin{array}{l}\text { ' } P \text { ' (compared } \\
\text { withcontrol) }\end{array}$ & \begin{tabular}{|c|} 
MEAN $\$$ SD \\
pcompared to \\
pre-ind values
\end{tabular} & $\begin{array}{l}\text { 'p' (compared } \\
\text { with control) }\end{array}$ & \begin{tabular}{|c|c|} 
MEAN $\$$ SD \\
p compared to \\
pre-ind values
\end{tabular} & $\begin{array}{l}\text { 'p' (compared } \\
\text { with control) }\end{array}$ \\
\hline $\begin{array}{l}\text { Pre-induction } \\
\end{array}$ & $116.2 \pm 4.213$ & $114.6 \pm 7.346$ & $\begin{array}{r}0.05 \\
\end{array}$ & $118.2 \pm 9.966$ & $>0.05$ & $119.4 \pm 8.897$ & $>0.05$ \\
\hline After induction & $\begin{array}{l}116.3 \pm 4.571 \\
p>0.05\end{array}$ & $\begin{array}{c}113.2 \pm 7.202 \\
p>0.05\end{array}$ & $>0.05$ & $\begin{array}{c}116.5 \pm 9.818 \\
p>0.05\end{array}$ & $>0.05$ & $\begin{array}{l}116.3 \pm 8.903 \\
p>0.05\end{array}$ & $>0.05$ \\
\hline Just after L\&1 & $\begin{array}{l}156.8 \pm 5.054 \\
p<0.001\end{array}$ & $\begin{array}{c}135.9 \pm 9.573 \\
p<0.001\end{array}$ & $<0.0001$ & $\begin{array}{c}140.6 \pm 13.4 \\
p<0.001\end{array}$ & $<0.0001$ & $\begin{array}{l}136+8.552 \\
p<0.001\end{array}$ & $<0.0001$ \\
\hline 1 min after $L \& I$ & $p<0.001$ & $\begin{array}{r}126.5+9.024 \\
p<0.001\end{array}$ & $<0.0001$ & $\begin{array}{r}129.3 \pm 13.08 \\
\mathrm{p}=0.001 \text { to }\end{array}$ & $<0.0001$ & $p<0.001$ & $<0.0001$ \\
\hline 3 min after $L \& \mid$ & $\begin{array}{r}135.8 \pm 3.727 \\
p<0.001\end{array}$ & $\mathrm{~ns}^{115.2 \pm 4.506}$ & $<0.0001$ & $\begin{array}{c}114.9 \pm 13.91 \\
p>0.05\end{array}$ & $<0.0001$ & $\begin{array}{c}113.6 \pm 12.11 \\
p>0.05\end{array}$ & $<0.0001$ \\
\hline $5 \mathrm{~min}$ after $L \& 1$ & $\begin{array}{l}\begin{array}{l}127.9 \pm 3.695 \\
p<0.001\end{array} \\
\end{array}$ & $\begin{array}{c}103.9 \pm 6.266 \\
p<0.001\end{array}$ & $<0.0001$ & $\begin{array}{c}113.2 \pm 11.13 \\
p>0.05\end{array}$ & $<0.0001$ & $\begin{array}{c}106+11.04 \\
p<0.001\end{array}$ & $<0.0001$ \\
\hline 10 min after L\&। & $\begin{array}{c}121.8 \pm 3.295 \\
p<0.001\end{array}$ & $\begin{array}{c}97.53 \pm 4.133 \\
p<0.001\end{array}$ & $<0.0001$ & $\begin{array}{r}107 \pm 13.48 \\
p=0.001 \text { to } 0.01\end{array}$ & $<0.0001$ & $\begin{array}{l}103.9+7.021 \\
p<0.001\end{array}$ & \\
\hline
\end{tabular}

Table 6 shows changes in the systolic blood pressure and comparison with baseline and control (Graph 5). 
$\underline{0}$

Table 5b: Changes In Systolic Blood Pressure: Comparison among the Study Drug Groups

\begin{tabular}{|l|l|l|l|}
\hline $\begin{array}{l}\text { SBP } \\
(\mathbf{m m} \text { Hg) } \\
\text { w.r.t time }\end{array}$ & $\begin{array}{l}\text { Group E vs L } \\
\text { P value }\end{array}$ & $\begin{array}{l}\text { Group L vs M } \\
\text { P value }\end{array}$ & $\begin{array}{l}\text { GroupM v } \\
\text { E } \\
\text { P value }\end{array}$ \\
\hline Pre-induction & $>0.05$ & $>0.05$ & $>0.05$ \\
\hline After induction & $>0.05$ & $>0.05$ & $>0.05$ \\
\hline Just after L \& I & $>0.05$ & $>0.05$ & $>0.05$ \\
\hline 1 min after L \& I & $>0.05$ & $>0.05$ & $>0.05$ \\
\hline 3 min after L\&I & $>0.05$ & $>0.05$ & $>0.05$ \\
\hline 5 min after L \&I & $<0.01$ & $>0.05$ & $>0.05$ \\
\hline 10 min after L\&I & $<0.01$ & $>0.05$ & $>0.05$ \\
\hline
\end{tabular}

[Table 5b] shows the comparison in SBP among the study drug groups [Figure 5]. Esmolol significantly attenuated $(\mathrm{P}<0.01)$ the $\mathrm{SBP}$ rise, $3 \mathrm{~min}$ and $5 \mathrm{~min}$ after L\&I in comparison to labetalol.

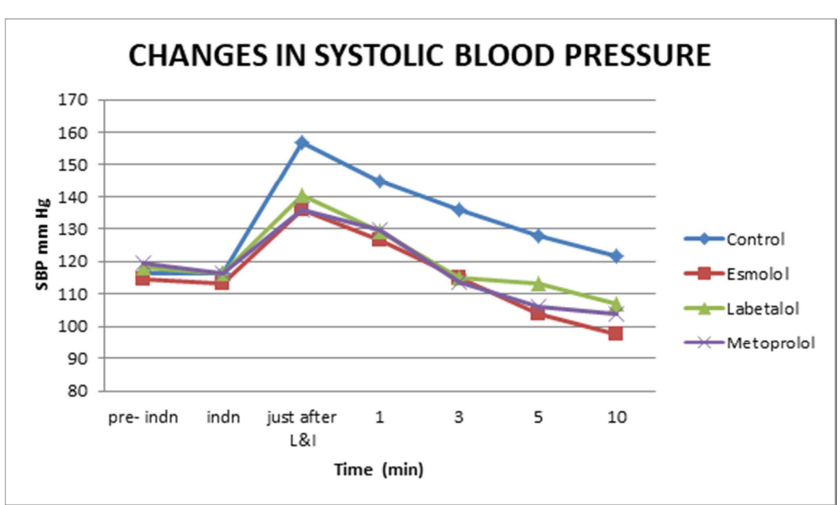

Figure 5: Changes in Systolic Blood Pressure.

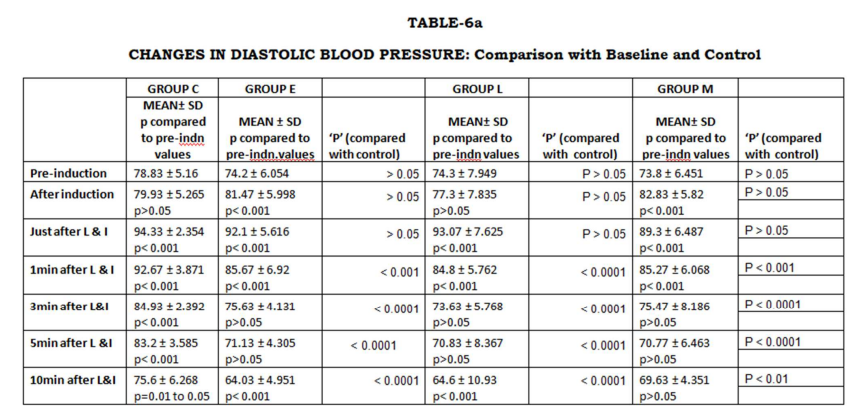

Table 6 shows changes in the diastolic blood pressure and comparison with baseline and control (Graph 6).

Table 6b: Changes In Diastolic Blood Pressure: Comparison among the Study Drug Groups

\begin{tabular}{|l|l|l|l|}
\hline $\begin{array}{l}\text { DBP } \\
(\mathbf{m m} \text { Hg) } \\
\text { w.r.t time }\end{array}$ & $\begin{array}{l}\text { Group E vs L } \\
\text { P value }\end{array}$ & $\begin{array}{l}\text { Group L vs M } \\
\text { P value }\end{array}$ & $\begin{array}{l}\text { GroupM v } \\
\text { E } \\
\text { P value }\end{array}$ \\
\hline Pre-induction & $>0.05$ & $>0.05$ & $>0.05$ \\
\hline After induction & $>0.05$ & $>0.05$ & $>0.05$ \\
\hline Just after L \& I & $>0.05$ & $>0.05$ & $>0.05$ \\
\hline 1 min after L \& I & $>0.05$ & $>0.05$ & $>0.05$ \\
\hline 3 min after L\&I & $>0.05$ & $>0.05$ & $>0.05$ \\
\hline 5 min after L \&I & $>0.05$ & $>0.05$ & $>0.05$ \\
\hline 10 min after L\&I & $>0.05$ & $>0.05$ & $<0.05$ \\
\hline
\end{tabular}

[Table 6b] shows comparison of DBP among the study drug groups [Figure 6]. No statistically significant difference was seen among the comparison in DBP between the study drug groups, except a statistically significant fall in esmolol compared to metoprolol at 10 minute following L\&I $(\mathrm{P}<0.05)$.

\section{CHANGES IN DIASTOLIC BLOOD PRESSURE}

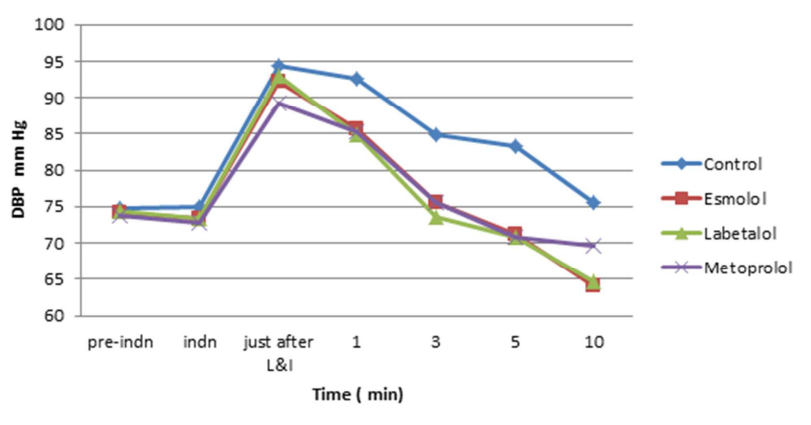

Figure 6: Changes in Diastolic Blood Pressure.

TABLE-7a

CHANGES IN MEAN BLOOD PRESSURE: Comparison with Baseline and Control

\begin{tabular}{|c|c|c|c|c|c|c|c|}
\hline & \multicolumn{2}{|c|}{ MEAN $5 D$} & \multicolumn{2}{|r|}{ MEAN土 SD } & \multirow[b]{2}{*}{$\begin{array}{l}\text { 'p' (compared } \\
\text { with control) }\end{array}$} & \multirow{2}{*}{\begin{tabular}{|c|} 
MEAN土 SD \\
GRouP M \\
pcompared to \\
pre-indn \\
values
\end{tabular}} & \multirow[b]{2}{*}{$\begin{array}{l}\text { 'p' (compared } \\
\text { with control) }\end{array}$} \\
\hline & 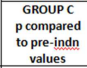 & \begin{tabular}{|c|}
$\begin{array}{c}\text { GROOPE } \\
\text { pcompared to } \\
\text { pre-indn } \\
\text { values }\end{array}$ \\
\end{tabular} & $\begin{array}{l}\text { 'p'(compared } \\
\text { with control) }\end{array}$ & $\begin{array}{l}\text { GRouPL } \\
\text { pcompered to } \\
\text { pre-indn } \\
\text { values }\end{array}$ & & & \\
\hline Pre-induction & $91.29 \pm 3.835$ & $87.68 \pm 6.1$ & $P>0.05$ & $88.92 \pm 7.454$ & $P>0.05$ & \begin{tabular}{|l|l|}
$89.01 \pm 5.2$ \\
\end{tabular} & $P>0.05$ \\
\hline Afterinduction & $\begin{array}{l}\begin{array}{l}92.04 \pm 3.915 \\
p>0.05\end{array} \\
p=0\end{array}$ & $\begin{array}{l}92.03 \pm 5.605 \\
p=0.01 \text { to } 0.05\end{array}$ & $P>0.05$ & $\begin{array}{l}90.36 \text { t7.615 } \\
p>0.05\end{array}$ & $P>0.05$ & $\begin{array}{l}94 \pm 5.981 \\
p=0.01 \text { to } 0.05\end{array}$ & $P>0.05$ \\
\hline Justafter L\&! & $\begin{array}{l}115.2 \pm 2.482 \\
p<0.001\end{array}$ & $\begin{array}{l}106.7 \pm 6.215 \\
p<0.001\end{array}$ & $P<0.0001$ & $\begin{array}{l}108.9 \pm 8.239 \\
p<0.001\end{array}$ & $P<0.01$ & $\begin{array}{l}104.9 \pm 6.104 \\
p<0.001\end{array}$ & $P<0.0001$ \\
\hline 1 min after $L \& 1$ & $\begin{array}{l}110.0112 .256 \\
p<0.001 \\
\text { p. }\end{array}$ & $\begin{array}{l}99.20877 .297 \\
p<0.001\end{array}$ & $P<0.0001$ & $\begin{array}{l}99.62 \text { t7.4.452 } \\
p<0.001\end{array}$ & $P<0.0001$ & $\begin{array}{l}10.00 .17 .349 \\
p<0.001\end{array}$ & $P<0.0001$ \\
\hline 3 min after L\&: & $\begin{array}{l}101.92 .2 .283 \\
p<0.001\end{array}$ & $\begin{array}{l}88.8223 .776 \\
p>0.05\end{array}$ & $P<0.0001$ & $\begin{array}{l}87.4 \pm 7.44 \\
p>0.05\end{array}$ & $P<0.0001$ & $\begin{array}{l}88.18 \div 9.039 \\
p>0.05\end{array}$ & $P<0.0001$ \\
\hline 5 min after L \& 1 & $\begin{array}{l}98.11 \pm 2.495 \\
p<0.001\end{array}$ & $\begin{array}{l}82.06 \pm 4.454 \\
p<0.001\end{array}$ & $P<0.0001$ & $\begin{array}{l}84.97 \pm 9.013 \\
n s\end{array}$ & $P<0.0001$ & $\begin{array}{l}82.51 \pm 7.356 \\
p=0.001 \text { to } 0.01\end{array}$ & $P<0.0001$ \\
\hline $10 \mathrm{~min}$ afterl\&! & $\begin{array}{r}91 \pm 4.03 \\
p>0.05\end{array}$ & $\begin{array}{r}75.2 \pm 4.348 \\
p<0.001\end{array}$ & $P<0.0001$ & $\begin{array}{l}78.73 \pm 11.17 \\
p<0.001\end{array}$ & $P<0.0001$ & \begin{tabular}{|l|}
$\begin{array}{l}81.04 \pm 4.662 \\
p<0.001\end{array}$ \\
$p$
\end{tabular} & $P<0.0001$ \\
\hline
\end{tabular}

Table 6 shows changes in the Mean blood pressure and comparison with baseline and control (Graph 7).

Table 7b: Changes in Mean Blood Pressure: Comparison among the Study Drug Groups

\begin{tabular}{|l|l|l|l|}
\hline $\begin{array}{l}\text { MBP } \\
\text { (mm Hg) } \\
\text { w.r.t time }\end{array}$ & $\begin{array}{l}\text { Group E vs L } \\
\text { P value }\end{array}$ & $\begin{array}{l}\text { Group L vs M } \\
\text { P value }\end{array}$ & $\begin{array}{l}\text { GroupM vs } \\
\text { E P value }\end{array}$ \\
\hline Pre-induction & $>0.05$ & $>0.05$ & $>0.05$ \\
\hline After induction & $>0.05$ & $>0.05$ & $>0.05$ \\
\hline Just after L \& I & $>0.05$ & $>0.05$ & $>0.05$ \\
\hline 1min after L \& I & $>0.05$ & $>0.05$ & $>0.05$ \\
\hline 3 min after L\&I & $>0.05$ & $>0.05$ & $>0.05$ \\
\hline 5 min after L \&I & $>0.05$ & $>0.05$ & $>0.05$ \\
\hline 10 min after L\&I & $>0.05$ & $>0.05$ & $<0.05$ \\
\hline
\end{tabular}

[Table 7b] shows comparison in MBP among the study drug groups [Figure 7]. No statistically significant difference was seen among the com-parison in MBP between the study drug groups, except a statistically signifi-cant fall in esmolol compared to metoprolol 10 minutes following L\&I $(\mathrm{P}<0.05)$.

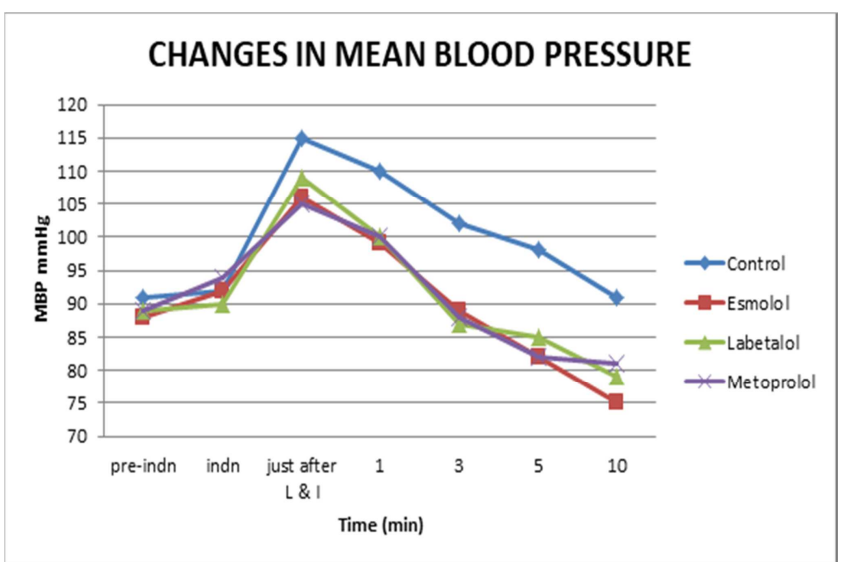

Figure 7: Changes in Mean Blood Pressure. 
TABLE-8a

CHANGES IN RATE PRESSURE PRODUCT: Comparison with Baseline and Control

\begin{tabular}{|c|c|c|c|c|c|c|c|}
\hline & \multicolumn{2}{|c|}{ MEAN 5 SD } & \multirow[b]{2}{*}{$\begin{array}{l}\text { 'p'lcompared } \\
\text { with controll) }\end{array}$} & \multirow[b]{2}{*}{\begin{tabular}{|c|} 
MEAN \pm SD \\
$\begin{array}{c}\text { GRROPPL } \\
\text { pcompared to } \\
\text { pre-indn } \\
\text { values }\end{array}$ \\
\end{tabular}} & \multirow[b]{2}{*}{ 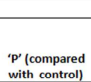 } & \multirow[b]{2}{*}{$\begin{array}{l}\text { MEAN } \text { SD } \\
\text { GRRoup M } \\
\text { pcompared to } \\
\text { pre-indn } \\
\text { values }\end{array}$} & \multirow[b]{2}{*}{$\begin{array}{l}\text { ' } \\
\text { with compartroll) }\end{array}$} \\
\hline & $\begin{array}{l}\text { GRoupC } \\
\text { p compered } \\
\text { to pre-indn } \\
\text { velues }\end{array}$ & 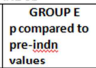 & & & & & \\
\hline Pre-induction & $9811 \pm 710.7$ & $10048 \pm 980.7$ & $P>0.05$ & $10191 \pm 1421$ & $P>0.05$ & $10516 \pm 1380$ & $P>0.05$ \\
\hline After induction & $\begin{array}{l}9816 \pm 752.5 \\
p>0.05\end{array}$ & $\begin{array}{l}9907 \pm 1247 \\
p>0.05\end{array}$ & $P>0.05$ & $\begin{array}{l}10044 \pm 1404 \\
p>0.05\end{array}$ & $P>0.05$ & $\begin{array}{l}10047 \pm 1185 \\
p>0.05\end{array}$ & $P>0.05$ \\
\hline Justafter L\&। & $\begin{array}{l}17479 \pm 1181 \\
p<0.001\end{array}$ & $\begin{array}{l}13978 \pm 1300 \\
p<0.001\end{array}$ & $P<0.0001$ & $\begin{array}{l}14282 \pm 2306 \\
p<0.001\end{array}$ & $P<0.0001$ & $\begin{array}{l}12649 \pm 1525 \\
p<0.001\end{array}$ & $P<0.0001$ \\
\hline 1 min after $L \& \mid$ & $\begin{array}{l}15761 \pm 934.6 \\
p<0.001\end{array}$ & $\begin{array}{l}12048 \pm 1236 \\
p<0.001\end{array}$ & $P<0.0001$ & $\begin{array}{l}13029 \pm 1802 \\
p<0.001\end{array}$ & $P<0.0001$ & $\begin{array}{l}11989 \pm 1501 \\
p<0.001\end{array}$ & $P<0.0001$ \\
\hline 3minafter L\&! & $\begin{array}{l}13959 \pm 657 \\
p<0.001\end{array}$ & \begin{tabular}{|l}
$11929 \pm 983.4$ \\
$p<0.001$
\end{tabular} & $P<0.0001$ & $\begin{array}{l}10992 \pm 1656 \\
p>0.05\end{array}$ & $P<0.0001$ & $\begin{array}{l}10423 \pm 1140 \\
p>0.05\end{array}$ & $P<0.0001$ \\
\hline 5 min after $L \& 1$ & $\begin{array}{l}12972 \pm 765.4 \\
p<0.001\end{array}$ & $\begin{array}{l}10537 \pm 1284 \\
p>0.05\end{array}$ & $P<0.0001$ & $\begin{array}{l}10793 \pm 1516 \\
p>0.05\end{array}$ & $P<0.0001$ & \begin{tabular}{|l}
$9600 \pm 1216$ \\
$p=0.01$ to 0.05
\end{tabular} & $P<0.0001$ \\
\hline $10 \mathrm{~min}$ afteri\&! & $\begin{array}{l}11551 \pm 730.1 \\
p<0.001\end{array}$ & \begin{tabular}{|l|}
$9310 \pm 996.4$ \\
$p>0.05$
\end{tabular} & $P<0.0001$ & $\begin{array}{l}9830 \pm 1569 \\
p>0.05\end{array}$ & $P<0.0001$ & $\begin{array}{l}8717 \pm 1296 \\
p<0.001\end{array}$ & $P<0.0001$ \\
\hline
\end{tabular}

Table 10 shows changes in the Rate pressure product and comparison with baseline and control (Graph 8).

Table 8b: Changes In Rate Pressure Product: Comparison among the Study Drug Groups

\begin{tabular}{|l|l|l|l|}
\hline $\begin{array}{l}\text { RPP } \\
\text { w.r.t time }\end{array}$ & $\begin{array}{l}\text { Group E vs L } \\
\text { P value }\end{array}$ & $\begin{array}{l}\text { Group L vs M } \\
\text { P value }\end{array}$ & $\begin{array}{l}\text { Group M vs } \\
\text { E P value }\end{array}$ \\
\hline Pre-induction & $>0.05$ & $>0.05$ & $>0.05$ \\
\hline After induction & $>0.05$ & $>0.05$ & $>0.05$ \\
\hline Just after L \& I & $>0.05$ & $<0.0001$ & $<0.01$ \\
\hline 1min after L \& I & $>0.05$ & $>0.05$ & $>0.05$ \\
\hline 3 min after L\&I & $>0.05$ & $>0.05$ & $<0.001$ \\
\hline 5 min after L \&I & $>0.05$ & $<0.05$ & $>0.05$ \\
\hline 10 min after L\&I & $>0.05$ & $<0.05$ & $>0.05$ \\
\hline
\end{tabular}

[Table 8b] shows comparison in RPP among the study drug groups [Figure 8]. Statistically significant lesser RPP was seen in metoprolol group com-pared to labetalol group just after L\&I $(\mathrm{P}<0.0001)$, at $5 \mathrm{~min}$ and at $10 \mathrm{~min}$ af-ter L\&I $(\mathrm{P}<0.5)$. Statistically significant lesser RPP was seen in metoprolol group compared to esmolol group just after L\&I $(\mathrm{P}<0.01)$ and at $3 \mathrm{~min}(\mathrm{P}<0.01)$ following $\mathrm{L} \& \mathrm{I}$.

\section{CHANGES IN RATE PRESSURE PRODUCT}

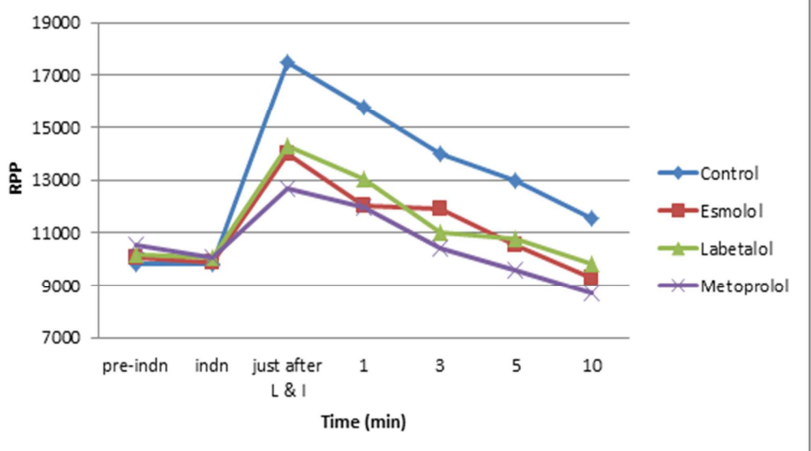

Figure 8: Changes in Rate Pressure Product.

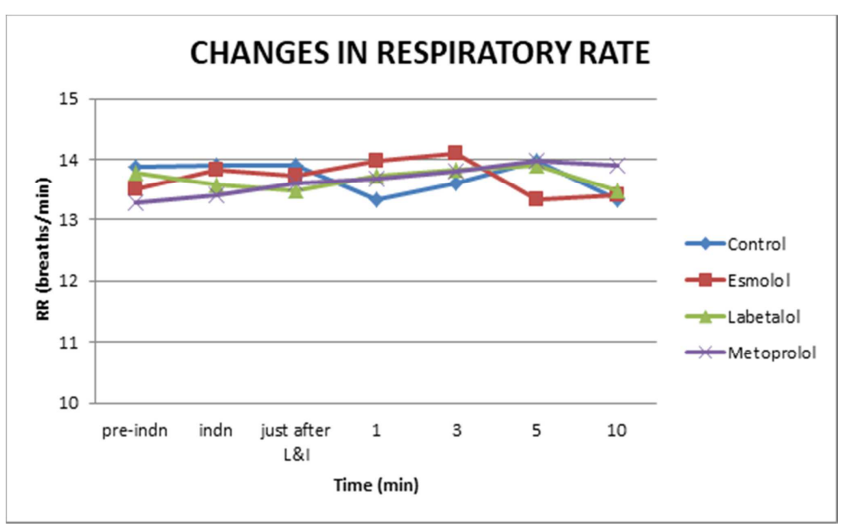

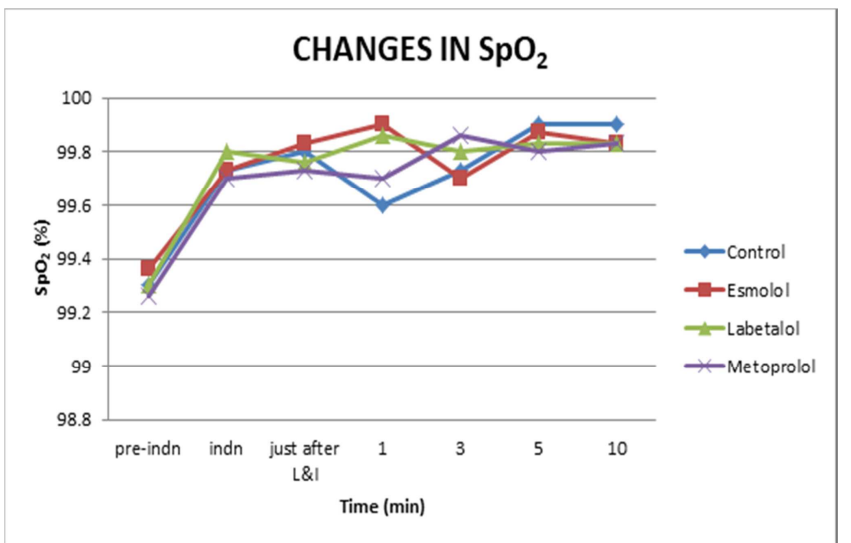

Figure 10: Changes in $\mathrm{SpO}_{2}$

Table 9: Incidence of Side Effects
\begin{tabular}{|l|l|l|l|l|l|}
\hline $\begin{array}{l}\text { S. } \\
\text { No. }\end{array}$ & Complications & $\begin{array}{l}\text { Group } \\
\text { C }\end{array}$ & $\begin{array}{l}\text { Group } \\
\text { E }\end{array}$ & $\begin{array}{l}\text { Group } \\
\text { L }\end{array}$ & $\begin{array}{l}\text { Group } \\
\mathbf{m}\end{array}$ \\
\cline { 3 - 6 } & No. (\%) & No. (\%) & No. $(\%)$ & No. $(\%)$ \\
\hline 1 & $\begin{array}{l}\text { Hypotension } \\
\text { (SBP<90mmHg) }\end{array}$ & $1(3.3 \%)$ & $\begin{array}{l}4 \\
(13.3 \%)\end{array}$ & $3(10 \%)$ & $\begin{array}{l}5 \\
(16.6 \%)\end{array}$ \\
\hline 2 & $\begin{array}{l}\text { Bradycardia } \\
\text { (HR<50bpm) }\end{array}$ & - & - & - & - \\
\hline 3 & $\begin{array}{l}\text { Sinus } \\
\text { tachycardia } \\
\text { (HR>120bpm) }\end{array}$ & $6(20 \%)$ & $3(10 \%)$ & 5 & $3(10 \%)$ \\
\hline 4 & Pain on injection & - & $1(3.3 \%)$ & - & - \\
\hline 5 & Ectopics & $3(10 \%)$ & - & $1(3.3 \%)$ & - \\
\hline 6 & Bronchospasm & - & - & - & - \\
\hline 7 & Miscellaneous & - & - & - & - \\
\hline
\end{tabular}

[Table 9] shows the incidence of complications in all the groups. Sinus tachycardia $(\mathrm{HR}>120 \mathrm{bpm})$ was seen in 6 patients $(20 \%), 3$ patients $(10 \%), 5$ patients $(16.6 \%)$ and 3patients (10\%) following L\&I in groups C, E, L and M respectively. Hypotension ( $\mathrm{SBP}<90 \mathrm{mmHg}$ ) was seen in one patient $(3.3 \%), 4$ patients $(13.3 \%), 3$ patients $(10 \%)$ and 5 patients $(16.6 \%)$ in groups $\mathrm{C}, \mathrm{E}, \mathrm{L}$ and $\mathrm{M}$ respectively. One patient developed pain on esmolol injection (3.3\%). No incidence of pain on injection was seen in other groups $(\mathrm{C}, \mathrm{L}$ $\& \mathrm{M})$. Three patients in control and one patient in labetalol group developed ectopic beats following L\&I which lasted for less than a minute and subsided without any intervention. No cases of ectopics were seen in esmolol and metoprolol groups. No other side effects attributable to the drug such as bronchospasm or bradycardia $(\mathrm{HR}<50 \mathrm{bpm})$ were noted.

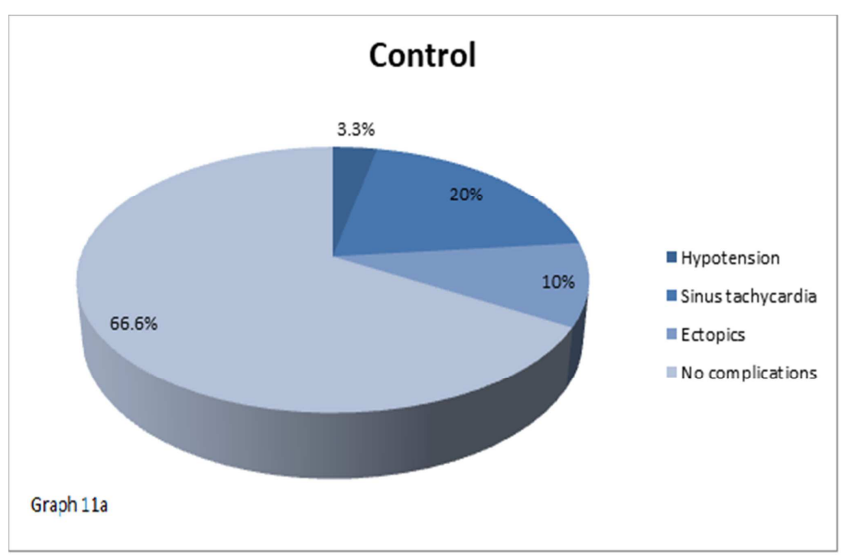




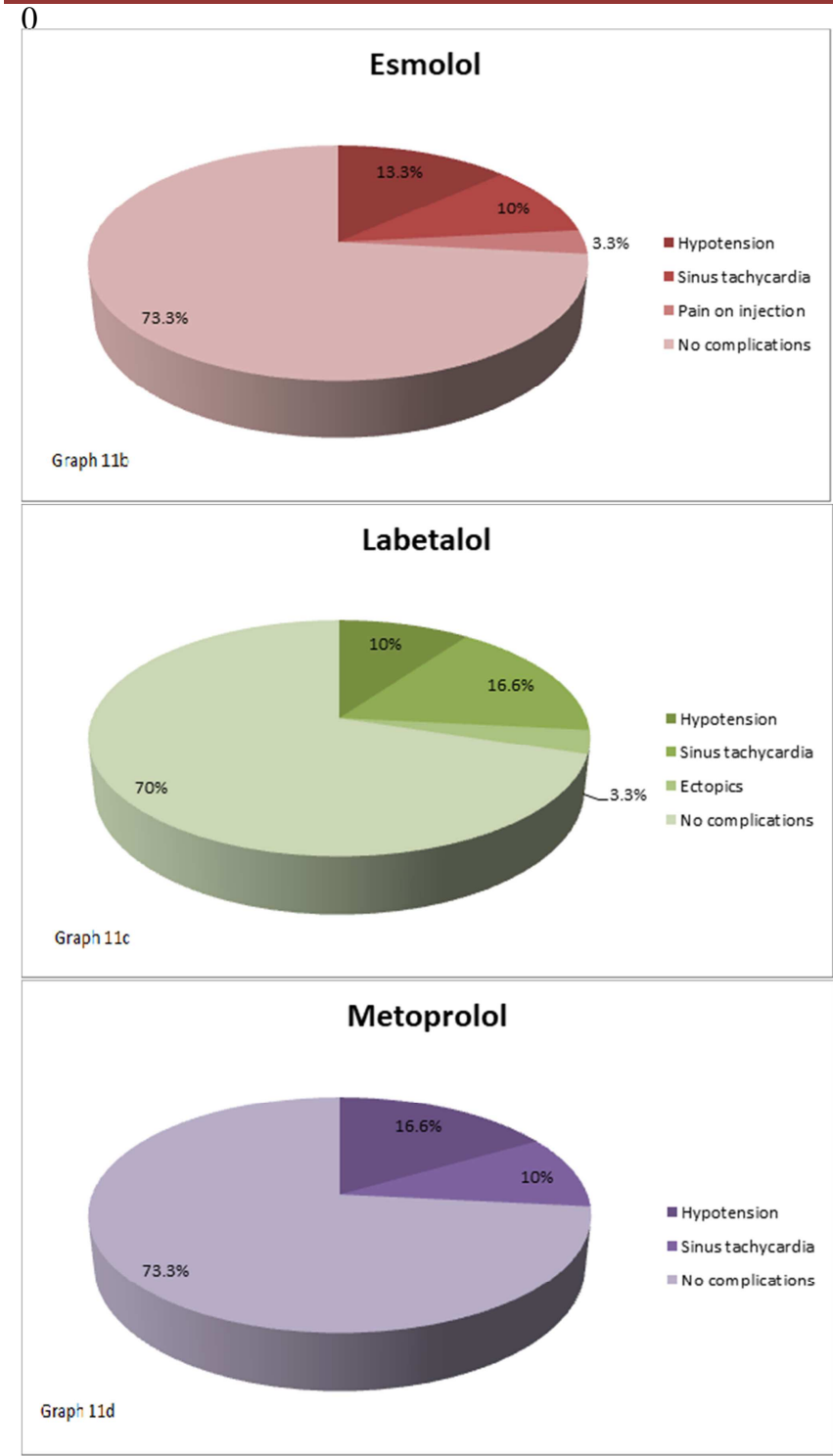

Figures 11a, 11b, 11c, 11d: Incidence of side effects.

\section{Discussion}

Each of the haemodynamic parameters were analysed by application of the significance of difference between means of groups.

1. Comparison with the baseline values was done by paired $t$ test within each group.

2. Comparison of each of the study drug group with the control group at their respective time intervals was done by ANOVA test (analysis of variance)

3. Comparison among the study drug groups at their respective time intervals was done by ANOVA test.

\section{Demofigureic profile:}

[Table 3a \& 3b; Figure -3a (i), 3a (ii) \& 3b]

The groups were comparable with respect to age, sex and weight.

\section{Changes in heart rate:}

[Table 4a \& 4b; Figure 4]

The pre-induction heart rate of the groups $\mathrm{C}, \mathrm{E}, \mathrm{L}$ and $\mathrm{M}$ were $84.43 \pm 5.37,87.77 \pm 7.27,86.2 \pm 9.71$ and $88 \pm 8.94(\mathrm{bpm})$ respectively and were comparable. In control, labetalol and metoprolol groups, the peak value of heart rate, seen just after L\&I, were 111.5 \pm 7.03, 101.6 $\pm 13.11,92.87 \pm 7.35$ respectively. In esmolol group, the peak value was seen at 3 min following L\&I (103.5 \pm 7.36$)$. In all the study drug groups, the rise in heart rate just after L\&I were significantly attenuated in comparison to control $(\mathrm{P}<0.01, \mathrm{P}<0.0001)$

\section{Comparison with baseline}

In control, esmolol and labetalol groups, the increase in the HR was statistically significant throughout the 10 minute study period compared to the pre-induction values $(\mathrm{p}<0.001)$. In metoprolol group, the increase in the HR was statistically significant only up to three min following L\&I $(p<0.01$ and $\mathrm{p}<0.05)$ after which, the rise in HR was statistically insignificant $(p>0.05)$ compared to the pre-induction values.

\section{Comparison with control}

In the esmolol and labetalol group, the increase in HR was statistically significant upto 1 minute $(\mathrm{P}<0.01)$ and 5 minute $(\mathrm{P}<0.05)$ respectively following $\mathrm{L} \& \mathrm{I}$ as compared to control. In metoprolol group, the increase in HR after L\&I, were significantly less than those in the control group $(\mathrm{P}<0 \bullet 001)$ at all times following L\&I.

\section{Comparison among the study drugs}

Statistically significant lesser HR rise was seen in esmolol group compared to labetalol group, at $1 \mathrm{~min}$ following L\&I $(\mathrm{P}<0.05)$. Statistically significant less $\mathrm{HR}$ rise was seen in metoprolol group compared to labetalol group just after L\&I $(\mathrm{P}<0.01)$, 1min $(\mathrm{P}<0.01)$ and $10 \mathrm{~min}(\mathrm{P}<0.01)$ following L\&I. Statistically significant less $H R$ rise was seen in metoprolol group compared to esmolol group, just after L\&I $(\mathrm{P}<0.001), 3 \mathrm{~min}(\mathrm{P}<0.0001), 5 \mathrm{~min}(\mathrm{P}<0.0001)$ and $10 \mathrm{~min}$ $(\mathrm{P}<0.0001)$ following $\mathrm{L} \& \mathrm{I}$.

The, the findings of our study in esmolol group are similar to those of Kasey P Bensky et al (2000), ${ }^{[15]}$ Rathore Arti et al (2002), ${ }^{[35]}$ Taner Tasyuz et al (2007), ${ }^{[4]}$ and Sarvesh P Singh et al (2010). ${ }^{[38]}$

The differences in our studies and other studies done on esmolol are likely due to the higher doses of esmolol used in their studies. We preferred lower doses of the study drugs so as to prevent any side effects.

The findings of our study in labetalol group are similar to those of Cope DHP et al (1979), ${ }^{[8]}$ Maharaj RJ et al $(1983),{ }^{[24]}$ Leslie John B et al (1989), ${ }^{[21]}$ and Castelli I et al (1995). ${ }^{[4]}$

Thus, the findings of our study in metoprolol group are similar to those of Zargar JA et al (2002), ${ }^{[50]}$ Liu Y et al $(2006),{ }^{[22]}$ and Coleman AJ et al (2007). ${ }^{[7]}$

\section{Changes In Systolic Blood Pressure:}

[Table 5a \& 5b; Figure 5].

In our study, the pre-induction SBP in all the groups were comparable. The pre-induction SBP of the groups C, E, L and $\mathrm{M}$ were $116.2 \pm 4.21,114.6 \pm 7.35,118.2 \pm 9.97$ and $119.4 \pm 8.89 \mathrm{mmHg}$ respectively. In all groups, the peak values of SBP seen just after intubation (L\&I) were $156.8 \pm 5.05,135.9 \pm 9.57,140.6 \pm 13.4$ and $136 \pm 8.55 \mathrm{mmHg}$ respectively. In all the study drug groups, the rise in SBP, just after laryngoscopy and intubation, was significantly 


\section{Comparison with baseline}

In control group, the increase in SBP was statistically significant throughout the 10 minute study period compared to the pre-induction values $(\mathrm{p}<0.001)$. In esmolol group, there was a statistically significant increase in SBP for $1 \mathrm{~min}$ following $\mathrm{L} \& \mathrm{I}(\mathrm{p}<0.001)$. At $3 \mathrm{~min}$, it was statistically insignificant $(\mathrm{p}>0.05)$. The SBP decreased further below the pre-induction values at $5 \mathrm{~min}$ and $10 \mathrm{~min}$ and this decrease was statistically significant $(\mathrm{p}<0.001)$. In labetalol group, the increase in SBP was statistically significant upto $1 \mathrm{~min}$ following L\&I. SBP remained statistically insignificant at $3 \mathrm{~min}$ and $5 \mathrm{~min}$. Thereafter, a statistically significant fall $(\mathrm{p}<0.001)$, was noted at $10 \mathrm{~min}$ following L\&I. In metoprolol group, the increase in SBP was statistically significant $(\mathrm{p}<0.001)$ one minute following L\&I compared to the pre-induction values. SBP decreased to values below the baseline at $3 \mathrm{~min}$ following L\&I, though the value was statistically insignificant. Thereafter, statistically significant fall $(\mathrm{p}<0.001)$ was noted at $5 \mathrm{~min}$ and $10 \mathrm{~min}$.

\section{Comparison with control}

Statistically significant fall in SBP $(\mathrm{P}<0.0001)$ was noted in all the study drug groups compared to control group at all times following L\&I.

\section{Comparison among the study drugs}

Statistically significant fall in SBP was noted in esmolol group compared to labetalol at $5 \mathrm{~min}$ and 10 minute following $\mathrm{L} \& \mathrm{I}(\mathrm{P}<0.01)$. All other comparison between the study drug groups were statistically insignificant $(\mathrm{P}>0.05)$.

The findings of our study in esmolol group are similar to those of Sheppard Shane et al (1990), ${ }^{[41]}$ Santosh Kumar et al (2003), ${ }^{[37]}$ and Taner Tasyuz et al (2007). ${ }^{[4]}$

The findings of our study in labetalol group are similar to those of Cope DHP et al (1979), ${ }^{[8]}$ Scott DB et al (1982), ${ }^{[39]}$ Castelli I et al (1995), ${ }^{[4]}$ and Sarvesh P Singh et al (2010). ${ }^{[38]}$ The findings of our study in metoprolol group are similar to those of Magnusson J et al (1986), ${ }^{[26]}$ Zargar JA et al $(2002){ }^{[50]}$ Liu Y et al (2006), ${ }^{[22]}$ and Coleman A.J et al (2007). ${ }^{[7]}$

\section{Changes In Diastolic Blood Pressure:}

[Table 6a \& 6b; Figure 6].

In our study, the pre-induction diastolic blood pressures in all the groups were comparable. The pre-induction DBP of the groups $\mathrm{C}, \mathrm{E}, \mathrm{L}$ and $\mathrm{M}$ were $74.983 \pm 5.16,74.2 \pm 6.05$, $74.3 \pm 7.95$ and $73.8 \pm 6.45 \mathrm{mmHg}$ respectively. The peak values of DBP, seen just after L\&I in all groups, were $94.33 \pm 2.35,92.1 \pm 5.61,93.07 \pm 7.63,89.3 \pm 6.49$ in groups $C$, $\mathrm{E}, \mathrm{L}$ and $\mathrm{M}$ respectively.

\section{Comparison with baseline}

In control group, the increase in DBP was statistically significant upto $5 \mathrm{~min}$ following $\mathrm{L} \& \mathrm{I}(\mathrm{p}<0.001)$ compared to pre-induction values. At the 10th minute a fall in DBP was noted which was statistically significant $(\mathrm{p}<0.05)$. In esmolol and labetalol group, the increase in DBP was statistically significant only upto one minute following $L \& I \quad(p<0.001)$ after which, a fall in DBP was noted. DBP at $3 \mathrm{~min}$ and $5 \mathrm{~min}$ was statistically insignificant $(\mathrm{p}>0.05)$. DBP at 10 minute following $L \& I$, in both the groups (E\&L), showed a statistically significant fall compared to pre-induction value $(p<0.0001)$. In metoprolol group, the rise in DBP was statistically significant upto 1minute following L\&I $(\mathrm{p}<0.0001)$. The values at 3 and 5 minutes were statistically insignificant $(\mathrm{p}>0.05)$. A fall in DBP was noted at the 10th minute following L\&I, though it was statistically insignificant $(\mathrm{p}>0.05)$.

\section{Comparison with control}

DBP values of all the study drug groups compared to control group showed statistically significant attenuation at all times following $\mathrm{L} \& \mathrm{I}(\mathrm{P}<0.01 ; \mathrm{P}<0.0001)$.

\section{Comparison among the study drugs}

No statistically significant difference was seen among the comparison in DBP between the study drugs, except a statistically significant fall in esmolol compared to metoprolol at 10 minute following $\mathrm{L} \& \mathrm{I}(\mathrm{P}<0.05)$.

The findings of our study in esmolol group are similar to those Santosh Kumar et al (2003), ${ }^{[37]}$ Taner Tasyuz et al (2007), ${ }^{[47]}$ and Sarvesh P Singh et al (2010). ${ }^{[38]}$

The findings of our study in labetalol group are similar to those of Maharaj RJ et al (1983), ${ }^{[24]}$ and Sarvesh P Singh et al (2010). ${ }^{[38]}$

\section{Changes in Mean Blood Pressure:}

[Table 7a \& 7b Figure 7]

In our study, the pre-induction mean blood pressures in all the groups were comparable. The pre-induction MBP readings of the groups $\mathrm{C}, \mathrm{E}, \mathrm{L}$ and $\mathrm{M}$ were $88.62 \pm 3.84$, $87.68 \pm 6.1,88.92 \pm 7.45$ and $89.01 \pm 5.2 \mathrm{mmHg}$ respectively. The peak value of MBP, seen just after L\&I in all groups, were $115.2 \pm 2.48,106.7 \pm 6.22,108.9 \pm 8.24,104.9 \pm 6.10$ in groups $\mathrm{C}, \mathrm{E}, \mathrm{L}$ and $\mathrm{M}$ respectively.

\section{Comparison with baseline}

In control group, the increase in $\mathrm{MBP}$ was statistically significant upto $5 \mathrm{~min}$ following $\mathrm{L} \& \mathrm{I}(\mathrm{p}<0.001)$ compared to pre-induction values. In esmolol group, the increase in MBP was statistically significant upto one minute following L\&I $(\mathrm{p}<0.001)$. MBP at $3 \mathrm{~min}$ was also found to be statistically insignificant $(p>0.05)$. Subsequently, a fall in MBP noted at $5 \mathrm{~min}$ and $10 \mathrm{~min}$ following L\&I, was found to be statistically significant $(\mathrm{p}<0.001)$. In labetalol group, the increase in MBP was statistically significant $(\mathrm{p}<0.0001)$ upto $1 \mathrm{~min}$ following L\&I. Later on the values at $3 \mathrm{~min}$ and $5 \mathrm{~min}$ were found to be statistically insignificant. Subsequently, the fall in MBP noted at 10th minute was found to be statistically significant $(\mathrm{p}<0.001)$. In metoprolol group, the MBP rise was statistically significant upto $1 \mathrm{~min}$ following $L \& I$ I $(p<0.001)$. The reading at $3 \mathrm{rd}$ minute was statistically insignificant. The fall in MBP noted, thereafter at 5th min and 10th min was found to be statistically significant $(p<0.01)$ compared to baseline.

\section{Comparison with control}

MBP values of all the study drug groups compared to control group showed statistically significant attenuation at all times following $\mathrm{L} \& \mathrm{I}(\mathrm{P}<0.0001)$. 


\section{Comparison among the study drugs}

No statistically significant differences were seen among the comparison in MBP between the study drug groups, except a statistically significant fall in esmolol compared to metoprolol at 10th minute following $\mathrm{L} \& \mathrm{I}(\mathrm{P}<0.05)$.

The findings of our study in esmolol group are similar to those of Menigaux C et al (2002), ${ }^{[27]}$ and Sarvesh P Singh et al (2010). ${ }^{[38]}$

The findings of our study in labetalol group are similar to those of Maharaj RJ et al (1983), ${ }^{[2]}$ and Sarvesh P Singh et al (2010). ${ }^{[38]}$

Liu Y et al (2006), ${ }^{[22]}$ and Coleman AJ et al (2007), ${ }^{[7]}$ found metoprolol effective in controlling the arterial pressure during L\&I. The findings in our study correlate these studies.

\section{Changes In Rate Pressure Product:}

[Table 8a \& 8b, Figure 8]

Rate Pressure Product is an index of myocardial oxygen consumption [Gobel FL11]. It is a product of systolic blood pressure and the heart. ${ }^{[11]}$ Rate pressure product exceeding 22,000 is commonly associated with myocardial ischaemia and angina [Robinson BF33]. Although RPP does not predict regional myocardial supply demand relationships, examination of the individual components (heart rate and SBP) is useful in the management of ischaemic heart disease [Kissin I20]. An increase in blood pressure without a change in heart rate appears to be better for myocardial oxygenation than an increase in HR along with increase in blood pressure [Moffitt E e29]

In our study, the pre-induction rate pressure products in all the groups were comparable. The pre-induction readings of RPP of the groups C, E, L and M were 9811 \pm 710.7 , $10048 \pm 980.7,10191 \pm 1421$ and $10516 \pm 1380$ respectively. The peak value of RPP seen just after laryngoscopy and intubation in all groups were $17479 \pm 1181,13978 \pm 1300$, $14282 \pm 2306,112649 \pm 1525$ in groups $\mathrm{C}, \mathrm{E}, \mathrm{L}$ and $\mathrm{M}$ respectively. RPP crossed the critical mark of 15000 in control group just after L\&I and at $1 \mathrm{~min}$ following L\&I. RPP in the study drug groups never crossed this value.

\section{Comparison with baseline}

In control group, the increase in RPP was statistically significant at all times following $L \& I(p<0.001)$ compared to pre-induction values. In esmolol group, the increase in RPP was statistically significant upto three minute following L\&I ( $\mathrm{p}<0.001)$. In labetalol group, the increase in RPP was statistically significant $(p<0.001)$ upto $1 \mathrm{~min}$ following $\mathrm{L} \& \mathrm{I}$. In metoprolol group, the increase in RPP was statistically significant $(\mathrm{p}<0.0001)$ upto $1 \mathrm{~min}$ following L\&I. RPP at 3rd min was statistically insignificant. A statistically significant $(\mathrm{p}<0.05)$ fall in RPP from the baseline was noted at $5 \mathrm{~min}$ and 10 min following L\&I.

\section{Comparison with control}

RPP values of all the study drugs compared to control showed statistically significant attenuation at all times following $\mathrm{L} \& \mathrm{I}(\mathrm{P}<0.0001)$.

\section{Comparison among the study drugs}

Statistically significant lesser RPP was seen in metoprolol group compared to labetalol group just after L\&I $(\mathrm{P}<0.0001)$, at $5 \mathrm{~min}$ and $10 \mathrm{~min}$ after L\&I. Statistically significant lesser RPP was seen in metoprolol group compared to esmolol group just after L\&I and at $3 \mathrm{~min}$ following L\&I.

The findings of our study in esmolol, labetalol and metoprolol group are similar to those of Rathore Arti et al (2002)35, Sarvesh P Singh et al (2010)38 and Zargar JA et al (2002)50 respectively.

\section{Changes In Respiratory Rate:}

[Table 9, Figure 9]

Comparison in the respiratory rate within a group and that among the study groups at their respective time intervals was statistically insignificant throughout the study period $(\mathrm{p}>0.05, \mathrm{P}>0.05)$.

\section{Changes in SpO2:}

[Table 10, Figure 10]

Comparison in the $\mathrm{SpO} 2$ within a group and that among the study groups at their respective time intervals was statistically insignificant throughout the study period $(\mathrm{p}>0.05, \mathrm{P}>0.05)$

\section{Incidence of Side Effects/Complications:}

[Table 11; Figure 11a, 11b, 11c \& 11d]

Sinus tachycardia was seen in 6 patients $(20 \%), 3$ patients (10\%), 5 patients $(16.6 \%)$ and 3patients (10\%) following L\&I in groups $\mathrm{C}, \mathrm{E}, \mathrm{L}$ and $\mathrm{M}$ respectively. Hypotension $(\mathrm{SBP}<90 \mathrm{mmHg})$ was seen in one patient $(3.3 \%), 4$ patients $(13.3 \%), 3$ patients $(10 \%)$ and 5 patients $(16.6 \%)$ in groups $\mathrm{C}, \mathrm{E}, \mathrm{L}$ and $\mathrm{M}$ respectively. One patient developed pain on esmolol injection $(3.3 \%)$. No incidence of pain on injection was seen in other groups $(\mathrm{C}, \mathrm{L} \& \mathrm{M})$. Three patients in control and one patient in labetalol group developed ectopic beats following L\&I which lasted for less than a minute and subsided without any intervention. No cases of ectopics were seen in esmolol and metoprolol groups. No other side effects attributable to the drug such as bronchospasm or bradycardia were noted.

Sheppard Shane et al (1990), ${ }^{[41]}$ noted pain on inj. in 1 patient $(\mathrm{n}=15)$ in both placebo and esmolol $100 \mathrm{mg}$.

Miller Donald R et al (1991), ${ }^{[28]}$ found hypotension the most common side effect. In E100 group, 25\% developed hypotension and $16 \%$ in placebo. Bradycardia in $1 \%$ patients and pain on inj. $1.6 \%$ in both esmolol and in placebo were noted.

Rathore Arti et al (2002), ${ }^{[35]}$ One patient (4\%) in esmolol 150 $\mathrm{mg}$ group developed bradycardia.

Sarvesh P Singh et al $(2010),{ }^{[38]}$ noted atrial ectopics in 1 patient in control (4\%) and one in esmolol (4\%) post intubation. 7 patients $(28 \%)$ in labetalol $0.25 \mathrm{mg} / \mathrm{kg}$ group developed bradycardia after study period. No cases of bradycardia were noted in our study.

Sharma Suman et al (1996), ${ }^{[40]}$ Kasey P Bensky et al (2000) ${ }^{[15]}$ Menigaux C et al (2002), ${ }^{[27]}$ Saif Ghaus M et al $(2002),{ }^{[36]}$ Tan PH et al (2002), ${ }^{[46]}$ Yutaka Oda et al $(2005),{ }^{[49]}$ and Taner Tasyuz et al $(2007),{ }^{[47]}$ did not notice any adverse reactions attributable to esmolol.

Scott DB et al (1982), ${ }^{[39]}$ noted that high doses of halothane (3\%) with labetalol predisposes to myocardial depressant effects of halothane and undesirable reduction in myocardial performance. 
0

Maharaj RJ et al (1983), ${ }^{[24]}$ No cardiac dysrhythmias were noted in the study with labetalol $(0.25 \mathrm{mg} / \mathrm{kg}$ and $0.5 \mathrm{mg} / \mathrm{kg})$. We noted premature ventricular contraction in one patient in labetalol group just after L\&I which persisted for less than $1 \mathrm{~min}$ and subsided without treatment.

Zargar JA et al (2002), ${ }^{[50]}$ noted sinus tachycardia of $55 \%$ in control and $20 \%$ in metoprolol $4 \mathrm{mg}$ group. PVC in $10 \%$ patients in control was seen 1 min after L\&I.

Liu Y et al (2006), ${ }^{[22]}$ noted that the incidence of bradycardia had no statistic difference between metoprolol group and placebo.

Coleman AJ et al (2007), ${ }^{[7]}$ noted cardiac rhythm disturbance of short duration of no apparent consequence in metoprolol group.

\section{Conclusion}

Our study demonstrates that metoprolol appreciably and remarkably atten-uated the cardiovascular stress response to laryngoscopy and intubation in comparison to esmolol and labetalol. Esmolol was comparably better than labetalol in attenuating this hemodynamic response.

Metoprolol can thus, be used as a safe and better alternative to esmolol and labetalol considering the favourable protective cardiovascular effects during laryngoscopy and intubation. Being a longer acting $\beta$-blocker in comparison to esmolol, it can also provide its protective effects even in the intra-operative period.

\section{References}

1. Alexander R, Binns J,Hetreed M. A controlled trial of the effects of esmo-lol on cardiac function. Br. J.Anaesth 1994; May 72(5):594-5.

2. Bruder N, Granthil C, Ortega D. Consequences and prevention methods of hemodynamic changes during laryngoscopy and intubation. Ann $\mathrm{Fr}$ Anaesth Reanim 1992; 11(1):57-71

3. Bikiye U, Ogurlu M, Gezer E. Effects of esmolol, lidocaine and fentanyl on haemodynamic responses to endotracheal intubation: A comparative study. Clinical Drug Investigation 2007:27:269-77

4. Castelli I, Steiner A, Kaufmann MA, Afille H, Schouten et al Comparative study of esmolol and labetalol to attenuate hyperdynamic states after Elec-troconvulsive therapy. Anaesth Analg. 1995; 80:557-61

5. Chia YY, Chan MH, Ko NH, Liu K. Role of $\beta$-blockade in anaesthesia and post operative pain management after hysterectomy. Br J. Anaesth. 2004; 93(6):799-805

6. Chung KS, Sinatra RS, Halevy JD, Paige D, Silverman DG. A Comparison of fentanyl,esmolol and their combination for blunting the haemodynamic responses during rapid sequence induction. Canadian Journal of Anaesthesia 1992; 39:774-9

7. Coleman AJ, Jordan C. Cardiovascular responses to Anaesthesia. Influence of $\beta$-adrenoreceptor blockade with metoprolol. Anaesthesia 1980(re-published online 2007; 34: 972-978

8. Cope DHP. Use of labetalol during halothane anaesthesia.Br J Clin.Pharmac. 1979; 8: 223S-227S

9. Ebert TJ, Bernstein JS, Stowe DF, Roerig D, Kampine JP. Attenuation of hemodynamic responses to rapid sequence induction and intubation in healthy patients with a single bolus dose of esmolol.J Clin Anesth. 1990:2:243-52

10. Forbes AM, Dally FG. Acute hypertension during induction of anaesthe-sia and endotracheal intubation in normotensive man. Br J.Anaesth. 1970; 42:618-624

11. Gobel FL, Nordstrom LA, Nelson RR, Jorgensen CR, Wang Y. The rate-pressure product is an index of myocardial oxygen consumption during ex-ercise in patients with angina pectoris. Circulation 1978; 57: $549-56$

12. Helfman SM, Gold MJ, DeLisser EA, Herrington CA. Which drug prevents tachycardia and hypertension associated with tracheal intubation:
Li-docaine, fentanyl or esmolol? Anaesthesia and Analgesia 1991; Apr;72(4):482-6

13. Helfman SM,Herrington CA and Gold MI. Bolus esmolol treatment for intraoperative tachycardia. Anaesth Anal. 1991; $\operatorname{Mar}(3): 220-3$

14. Inada E, Cullen DJ, Nemeskal R, Teplick R. Effect of labetalol on the hemodynamic response to intubation. J Clin Anesth. 1989;4:11-5

15. Kasey P Bensky, Linda Donahue Spencer, G Erik Hertz, Martha T Anderson, Robert James. The dose related effects of bolus esmolol on heart rate and BP following L\&I. AANA Journal October 2000; Vol68 No5 $: 437-441$

16. Kamra S, Wig J, Sapru RP. Topical nitroglycerine.A safeguard against pressor response to tracheal intubation. Anaesthesia 1986;41:1087-91

17. Kindler CH, Schumacher PG, Schneider MC, Urwyler A. Effects of intra-venous lidocaine and/or esmolol on haemodynamic responses to laryngos-copy and tracheal intubation: a double blind controlled clinical trial. Journal of Clinical Anaesthesia 1996; Sept; 8(6): 491-6

18. King BD, Harris LC, Griefenestein FE, Elder JD, Dripps RD. Reflex cir-culatory response to direct laryngoscopy and intubation performed during general anaesthesia. Anaesthesiology 1951; 12:556

19. Kim HY, Chung CW, Lee HY, Yim. The effect of labetalol on the hemo-dynamic response to endotracheal intubation. Korean J Anesthesiol 1994; 27:1611-9

20. Kissin I, Reves JG, Mardis M. Is the rate pressure product a misleading guide? Anaesthesiology 1980; 52: 373-4

21. Leslie John B, Kalayjian RW, Mc Loughlin TM, Plachetka JR. Attenuation of hemodynamic response to endotracheal intubation with preinduction intravenous labetalol. J Clinical Anaesth 1989;1:194-200

22. Liu Y, Huang CL, Zhang LN, Cai HW, He M, Guo QL. Influences of peri-operative metoprolol on hemodynamics and myocardial ischaemia in elderly patients undergoing non-cardiac surgery. Zhong Nan Da Xue Xue Bao Yi Xue Ban. 2006 Apr;31(2):249-53

23. Liu Philips et al. Esmolol for control of increase in heart rate and blood pressure during tracheal intubation after thiopentone and succinylcholine. Can J. Soc. 33; 5:556-62

24. Maharaj RJ, Thompson M, Brock JG, Williamson R, Drowing JW. Treatment of hypertension following endotracheal intubation.A study com-paring the efficacy of labetalol, practolol and placebo. S Afr Med J 1983;63:691-4

25. Magnusson J, Werner O, Carlsson C,Pettersson KI, Nrden N.Metoprolol, fentanyl and stess response to microlaryngoscopy. Effect on arterial pres-sure, heart rate and plama concentration of catecholamine, ACTH and corti-sol. Br. J Anaesth 1983:55:405-14

26. Magnusson J, Thulin T, Werner O, Jarhult J,Thomson D.Haemodynamic Effects Of Pretreatment With Metoprolol In Hypertensive Patients Undergoing Surgery.Br J Anaesth 1986;58(3) $251-260$

27. Menigaux C, Guinard B, Adam F et al. Esmolol prevents movement and attenuates the BIS response to orotracheal intubation. Br J Anaesth. 2002;89:852-62

28. Miller Donald R., Martineau R.J., et al: Bolus administration of esmolol in controlling the hemodynamic response to tracheal intubation. The Cana-dian Multicentre Trial. Can J. Anaesthesia 1991.

29. Moffitt E, Sethna D, Bussell J, Raymond M, Matloff J, Gray R. Hemodynamics and myocardial metabolism after acute $\beta$-adrenergic blockade in coronary patients. Anesth Analg 1984; 63: 540-1

30. Nahid Aghdaii, Azarfarin R,Yazdanian F, Faritus S Z.Cardiovascular responsed to orotracheal intubation in patients undergoing coronary artery bypass grafting surgery. MEJ Anesth 2010;20(6):833-837

31. Prys Roberts C, Melache R \& Foex P (1971). Studies of anaesthesia in relation to hypertension.Cardiovascular responses of treated and untreated patients. Br. J. Anasth.1971;43:122-137

32. Prys Roberts, Foex P, Biro G: Studies of anaesthesia in relation to hypertension versus adrenergic beta blockade. Brit J Anaesthesia 1979;45: 671

33. Robinson BF, Relation of heart rate and systolic blood pressure to the onset of pain in angina pectoris. Circulation 1967;35:1073-83

34. Ramanathan J, Sibai BM, Madie WC, Chauhan D, Riuz AG. The use of labetalol for attenuation of hypertensive responses to endotracheal intuba-tion on pre-eclampsia. AM J Obstet Gynecol 1988; 159:650-4

35. Rathore A, Gupta HK, Tanwar GL. Attenuation of the pressor response to laryngoscopy and endotracheal intubation with different doses of esmolol. Indian J Anaesth 2002;46:449-52

36. Saif GhausM, Vinitha Singh, Kumar A, Wahal R, Bhatia VK, Agrawal J A study of cardiovascular response during laryngoscopy and intubation by ultra-short acting b-blocker Esmolol. Indian J. Anaesth 2002; 46(2):104-106 
37. Santosh Kumar, Mishra MN, Mishra LS, Sapna Bathla. Comparative study of efficacy of i.v esmolol, diltiazem and magnesium sulphate in atten-uating haemodynamic response to laryngoscopy and intubation.Indian J. Anaesth 2003;47(1):41-44

38. Sarvesh P Singh, Abdul Quadir, Poonam Malhotra. Comparison of esmolol and labetalol, in low doses, for attenuation of sympathomimetic re-sponse to laryngoscopy and intubation. Saudi J. Anaesth 2010; Vol4;issue3:163-168

39. Scott DB, Buckley, F.P., Littlewood, D.G.,Macrae,W.R., Arthur. G.B. Circulatory effects of labetalol during halothane anaesthesia. Anaesthesia 1982;33:145-156

40. Sharma Suman, Mitra S, Grover VK, Kalra R. Esmolol blunts the haemodynamic responses to tracheal intubation in treated hypertensive patients. Candian Journal of Anaesthesia 1996 Aug; 43 (8): 778-82

41. Sheppard Shane, Eagle CJ, Strunon L. A bolus dose of esmolol attenuates tachycardia and hypertension after tracheal intubation. Canadian Journal of Anaesthesia 1990;37:202-205

42. Singh H, Vichitvejpaisal P, Gaines GY, White PF. Comparative effects of lidocaine, esmolol and nitroglycerin in modifying the haemodynamic re-sponses to laryngoscopy and intubation. Journal of Clinical Anaesthesia 1995; Feb 7(1):5-8

43. Stoelting RK, Peterson C. Circulatory changes during anesthetic induction: impact of d-tubocurarine pre-treatment, thiamylal, succinylcholine, laryngoscopy and tracheal lidocaine. Anesth Analg(Cleve) 1976; 55:77-
44. Stoelting RK. Blood pressure and heart rate changes during short duration laryngoscopy for tracheal intubation. Influence of viscous or intrave-nous lidocaine. Anaesth Analg. 1978; 57:197

45. Stoelting RK. Attenuation of blood pressure response to laryngoscopy and tracheal intubation with sodium nitroprusside. Anesth Analg 1979;58:116-9

46. Tan P H, Yang HC, Lin CR, Lan KC, Chen CS: Combined use of esmolol and nicaridipine to blunt the haemodynamic changes following laryngscopy and tracheal intubation:Anaesthesia 2002, 57, 1207-1211

47. Taner Tasyuz, Ismet Topcu, Sabri Ozaslan, Melek Sakarya: Effects of esmolol on hemodynamic responses to L\&I in diabetic vs Non-Diabetic pa-tients. Turk J Med Sci 2007;37(5);289-296

48. Yuan L, Chia YY, Jan KT et al. The effect of single bolus dose of esmolol for controlling the tachycardia and hypertension during laryngoscopy and tracheal intubation. Acta Analgesiologica Sinica 1994; Sep; 32(3): 147-52

49. Yutaka Oda, Kiyonobu Nishikawa, Ichiro Hase, Akira Asada. The short acting $\beta 1$-adrenoreceptor antagonists Esmolol and landiolol supresss the bispectral index response to tracheal intubation during sevflurane anaes-thesia. Anaesth Analg 2005;100:733-7

50. Zargar JA, Naqash IA, Gurcoo SA, Mehraj-ud-din. Effect of metoprolol and esmolol on rate pressure product and ECG changes during laryngosco-py and tracheal intubation in controlled hypertensive patients. Indian J. Anaesth 2002; 46:365-8

Copyright: (C) the author(s), publisher. Academia Anesthesiologica International is an Official Publication of "Society for Health Care \& Research Development". It is an open-access article distributed under the terms of the Creative Commons Attribution Non-Commercial License, which permits unrestricted non-commercial use, distribution, and reproduction in any medium, provided the original work is properly cited.

How to cite this article: Deepak R, Lalwani J, Shah PJ, Dubey KP. Effect of Esmolol, Labetalol and Metoprolol for Attenuating the Cardiovascular Stress Response to Laryngoscopy and Intubation: A Comparative Study. Acad. Anesthesiol. Int. 2019;4(2):91-101.

DOI: dx.doi.org/10.21276/aan.2019.4.2.24

Source of Support: Nil, Conflict of Interest: None declared. 